\title{
Effect of Pre-exposure Use of Amantadine on COVID-19 Infection: A Hospital-Based Cohort Study in Patients With Parkinson's Disease or Multiple Sclerosis
}

\author{
Walaa A. Kamel ${ }^{1,2 *}$, Mohmed I. Kamel ${ }^{3}$, Almunther Alhasawi ${ }^{4}$, Sameh Elmasry ${ }^{4}$, \\ Fajer AlHamdan ${ }^{5}$ and Jasem Y. Al-Hashel ${ }^{2,6}$
}

${ }^{1}$ Neurology Department, Faculty of Medicine, Beni-Suef University, Beni-Suef, Egypt, ${ }^{2}$ Neurology Department, Ibn-Sina Hospital, Kuwait City, Kuwait, ${ }^{3}$ Occupational and Environmental Medicine, Alexandria University, Alexandria, Egypt, ${ }^{4}$ Internal Medicine and Infectious Diseases Consultant, Infectious Disease Hospital, Kuwait City, Kuwait, ${ }^{5}$ Internal Medicine Department, Al-Sabah Hospital, Kuwait City, Kuwait, ${ }^{6}$ Department of Medicine, Faculty of Medicine, Kuwait University, Kuwait City, Kuwait

\section{OPEN ACCESS}

Edited by:

Sara Palermo,

Carlo Besta Neurological Institute (IRCCS), Italy

Reviewed by:

Abdul Mannan Baig, Aga Khan University, Pakistan

Sandra Paulina Smieszek, Vanda Pharmaceuticals Inc., United States

${ }^{*}$ Correspondence: Walaa A. Kamel walaaneuro@yahoo.com

Specialty section

This article was submitted to

Neuroinfectious Diseases,

a section of the journal

Frontiers in Neurology

Received: 01 May 2021 Accepted: 07 September 2021 Published: 07 October 2021

Citation:

Kamel WA, Kamel MI, Alhasawi A,

Elmasry S, AlHamdan F and

Al-Hashel JY (2021) Effect of

Pre-exposure Use of Amantadine on COVID-19 Infection: A Hospital-Based Cohort Study in Patients With Parkinson's Disease or Multiple

Sclerosis. Front. Neurol. 12:704186.

doi: 10.3389/fneur.2021.704186
Background: Amantadine has been proposed to inhibit E-channel conductance in reconstituted lipid bilayers of severe acute respiratory syndrome coronavirus 2 (SARS-CoV-2). We aimed to study whether patients on amantadine have altered risks of contracting COVID-19 infection.

Methods: We conducted a hospital-based, observational, retrospective cohort study using data for patients on amantadine supported by data given by the patients through an online questionnaire. We included registered amantadine users in our hospital for 6 months or more on March 1, 2020, and non-amantadine users to act as the control group. We used forced entry, multiple logistic regression models to estimate adjusted ORs for amantadine adjusting for the confounders.

Findings: Between September 1, 2019, and March 1, 2020, 212 patients with Parkinson's disease (PD) or multiple sclerosis (MS) received greater than one equal to two prescriptions of amantadine. We selected a random sample of diagnoses which matched 424 patients of non-amantadine users (1:2) as a control group (424 patients). Between March 1, 2020, and March 1, 2021, 256 patients responded to our online questionnaire, 87 patients were on amantadine (group I), and 169 patients were not (control group, group II). COVID-19 disease infection proved to be 5.7 and $11.8 \%$ in group I and II patients, respectively. Increased odds of COVID-19 in multivariable-adjusted models were associated with old age and history of contact with COVID cases. Amantadine was associated with a significantly reduced risk of COVID-19 disease infection (adjusted OR $0.256,95 \% \mathrm{Cl} 0.074-0.888)$.

Interpretation: Amantadine is associated with a reduced risk of COVID-19 infection after adjusting for a broad range of variables. History of contact with COVID cases and old age are risk factors for COVID-19 infection. Therefore, we recommended randomized clinical trials investigating amantadine use for the prevention of COVID-19.

Keywords: Amantadine, pre-exposure, COVID-19, infection, multiple sclerosis, Parkinson's disease 


\section{INTRODUCTION}

Antiviral properties for amantadine were initially reported and first used to treat influenza A in 1963 and approved as a prophylactic treatment in 1976 against the influenza virus A (1). Due to the high resistance of amantadine, is not recommended for the treatment of influenza A (2). Amantadine was tried for the treatment of different neurological diseases and approved by the Food Drug Administration (FDA) to treat levodopainduced dyskinesia (LID) in Parkinson's disease (PD) (3). Offlabel uses include improving fatigue in multiple sclerosis (MS) (4) and improving arousal following a brain injury. At the time of the severe acute respiratory syndrome (SARS) epidemic in 2002, amantadine was studied (in vitro) and showed therapeutic potential (5).

Recognition of modest antiviral effects shown for adamantanes led to the suggestion that it could be repurposed for COVID-19 $(6,7)$. This was supported by the fact that patients on this antiviral drug were serologically negative after being in contact with positive cases. Moreover, those who tested positive did not develop the symptoms caused by the virus (8).

Many theories explained the antiviral mechanism as a lipophilic molecule that traverses the lysosome membrane. Amantadine acts as an alkalizing agent that hinders viral ribo nucleic acid (RNA) release into the cell (9).

It was hypothesized that amantadine would give a superior outcome due to decreased infectivity and replication of the virus, based on the fact that amantadine significantly downregulated expression cathepsin L (CTSL) gene coding for the CTSL and lysosomal protease involved in severe acute respiratory syndrome coronavirus 2 (SARS-CoV-2) human cell entry (10). Clinical studies are needed to examine the therapeutic utility of amantadine in COVID-19 infection.

Evaluations of the effectiveness of amantadine use for the prevention of SARS-CoV-2 infection are lacking. In this hospital-based cohort study, we aimed to investigate if ongoing amantadine use in the $6 \mathrm{~m}$ before March 1, 2020, considered the start of the outbreak in Kuwait (i.e., analogous to prophylactic use before exposure) was associated with a lesser risk of contracting COVID-19 infection. As a result of understanding the associations between medications and improved outcomes, clinical trials could provide a base for further insights into disease mechanisms, pathogenesis, and possible preventive measures if confirmed from cohort studies.

\section{METHODS}

\section{Data Source and Study Population}

We conducted a retrospective cohort study using electronic health record data from hospital care practices focusing on patients with the diagnosis of PD and MS who were compliant on their usual medications for at least 6 months before enrollment,

Abbreviations: SARS-CoV-2, severe acute respiratory syndrome coronavirus 2; PD, Parkinson's disease; MS, multiple sclerosis; FDA, food drug administration; LID, levodopa-induced dyskinesia; RNA, ribo nucleic acid; CTSL, cathepsin L; PCR, polymerase chain reaction; ICU, intensive care unit; SD, standard deviations; $\mathrm{HR}$, hazard ratio; $\mathrm{CI}$, confidence interval; $\mathrm{OR}$, odds ratio; PCR, polymerase chain reaction. with information on age, sex, and medications. Institutional Review Board approval was obtained from our hospital and the ministry of health (Figure 1).

\section{Inclusion Criteria}

All are 16 years old or above.

\section{Patients}

We selected participants from Parkinson's disease and multiple sclerosis patients who attended the outpatient clinic. Patients on amantadine acted as a case group and non-amantadine users as a control group.

\section{Exclusion Criteria}

- Patients who were not regularly on amantadine in the last 6 months before enrollment in the study.

- Recent change in his medications (previous 6 months).

- Patients outside Kuwait during the COVID-19 pandemic period (difficult to get their Polymerase Chain Reaction (PCR) results).

- MS patients who recently (in the past year) received glucocorticoids that may increase the risk of contracting infections (11), including COVID-19 (12).

We divided patients according to their current treatment into two groups; amantadine-user group (group I) $(n=212)$ and a sample of patients with randomly selected diagnoses matched non-amantadine users group II, with the proportion of 1:2.

\section{Survey Assessments}

Participants answered an online questionnaire (created by W. AK.). Surveys queried about; (1) amantadine exposure and dose, (2) COVID-19-related symptoms, (3) testing status and outcomes, (4) comorbid conditions, (5) smoking status, (6) employment, (7) personal and household contact, (8) social distancing practices, (9) mask use, history of travel, (10) COVID19 infection symptoms (as; fever, respiratory symptoms, catarrhal symptoms), (11) the result of COVID-19 PCR in case of infection, (12) symptoms indicating severe infection (such as; need for $\mathrm{O} 2$ use, hospitalization, intensive care unit (ICU) admission, or ventilatory need), and (13) the effect of infection on main neurologic disease, mood, and memory. Results of COVID19 PCR testing of the respondents were blindly retrieved using the national COVID-19 database (M.WH.).

\section{Data Collection}

We collected data on socio-demographic characteristics, chronic diseases, medications, COVID 19 risk factors, the severity of symptoms, swab results, and sequence of COVID infection on different neurologic symptoms.

\section{Statistical Analysis}

We presented continuous variables as either the means \pm standard deviations (SDs) or the medians (with interquartile ranges). For categorical variables, we calculated the frequency rates and percentages of patients in each category. We compared continuous variables using independent group $t$-tests in normally distributed data. Otherwise, we used the Mann-Whitney test. We compared the proportions of categorical variables using 


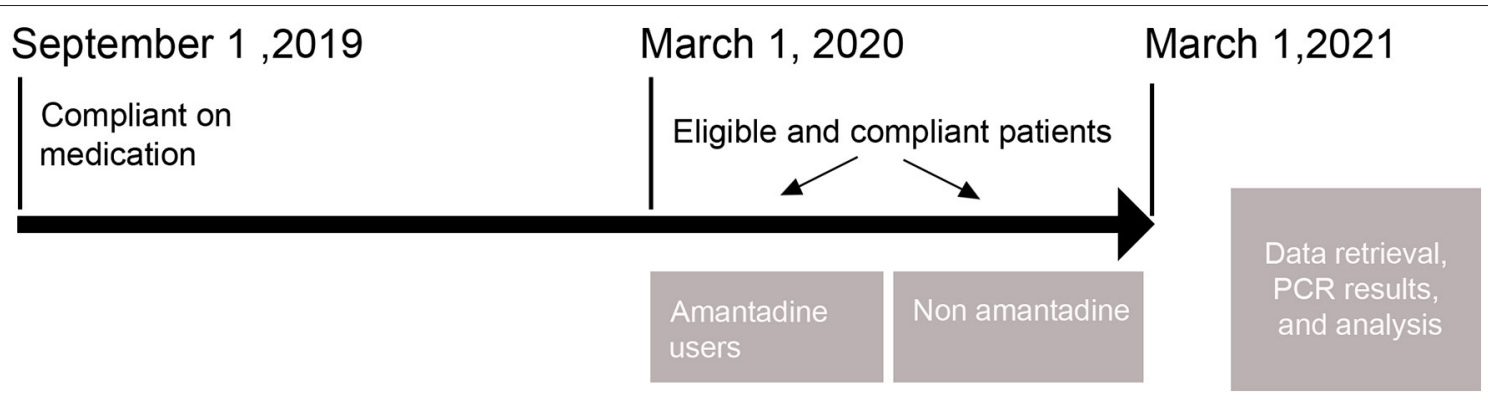

FIGURE 1 | Study diagram.

\section{Study Profile}

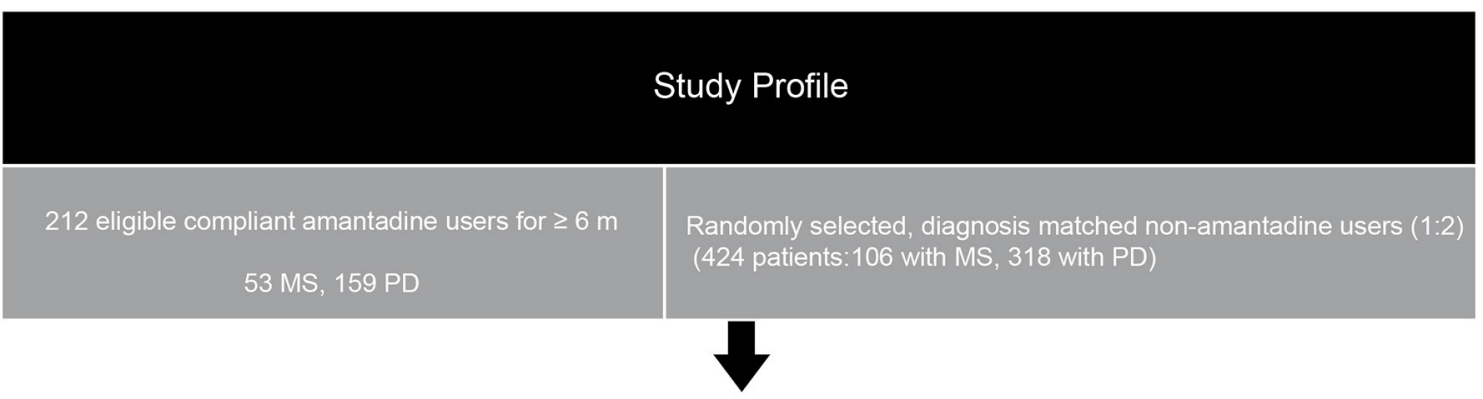

\section{Questionnaire link}

was sent for total 636 patients

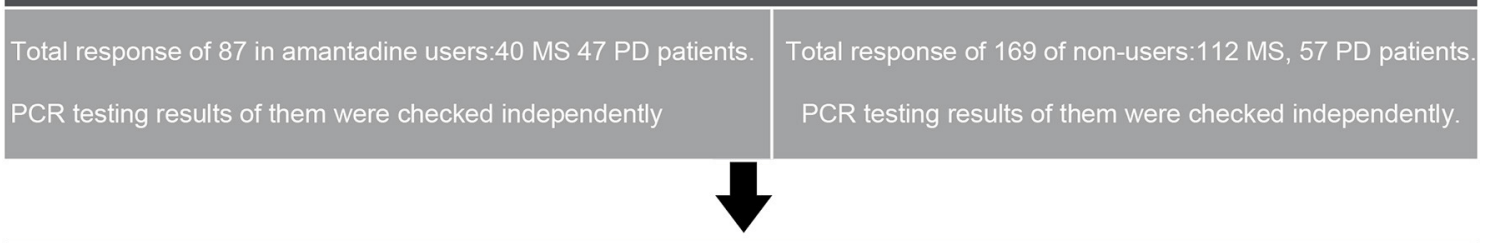

Total 256 PCR testing were independently retrieved

5 total amantadine users were diagnosed with COVID -19 infection,one with MS,4 with PD

20 total non-amantadine users were diagnosed with COVID -19 infection ,11 MS,9 with PD.

FIGURE 2 | Study profile.

the $\chi^{2}$-test, although we used Fisher's exact test when the data were limited. Multiple logistic regression analysis was applied to determine the prognostic factors, and the odds ratio (OR) with $95 \%$ confidence interval $(95 \%$ CI) was reported. Statistical analyses were performed using SPSS 21.

\section{RESULTS}

\section{Overall Study Population}

The study design is shown in Figure 1. Two hundred twelve patients were identified (with the diagnosis of PD or MS) to be compliant on amantadine for at least 6 months before
March 1, 2020 (i.e., index date). A group of patients with randomly selected diagnoses matched the number of $\mathrm{PD}$, MS patients who were not on amantadine. After sending the questionnaire link to patients, we got 256 responses in both groups (Figures 1, 2).

\section{Presenting Characteristics}

We included 256 patients in our analysis: 87 patients were amantadine users, and 169 were non-users. The results showed that the mean age \pm SD of group I was higher than that of group II $(52.5 \pm 12.4[31,75]$ vs. $46.9 \pm 16.2[16,84])$. Amantadine users are more likely to be men (50.6\% of users were women; $56.2 \%$ 
TABLE 1 | Socio-demographic characteristics of both groups.

\begin{tabular}{|c|c|c|c|c|}
\hline Characteristic & $\begin{array}{c}\text { Group I } \\
(N, \%) \\
87\end{array}$ & $\begin{array}{c}\text { Group II } \\
(N, \%) \\
169\end{array}$ & $\begin{array}{c}\text { Total } \\
(n=256)\end{array}$ & $P$ \\
\hline Parkinson's disease & $47(54)$ & $57(33.7)$ & $104(40.6)$ & \\
\hline Multiple sclerosis & $40(46)$ & $112(66.3)$ & $154(59.4)$ & \\
\hline \multicolumn{5}{|l|}{ Age, 10 years } \\
\hline $20-29$ & $0(0.0)$ & $22(13.0)$ & $22(8.6)$ & $0.003^{*}$ \\
\hline 30-39 & $14(16.1)$ & 45 (26.6) & $59(23)$ & \\
\hline $40-49$ & $23(26.4)$ & $36(21.3)$ & $59(23)$ & \\
\hline $50-59$ & $23(26.4)$ & $26(15.4)$ & $49(19.1)$ & \\
\hline $60+$ & $27(31.0)$ & $40(23.7)$ & $67(26.2)$ & \\
\hline Mean \pm SD & $\begin{array}{c}52.48 \pm 12.39 \\
(31,75)\end{array}$ & $\begin{array}{c}46.93 \pm 16.18 \\
(16,84)\end{array}$ & & \\
\hline \multicolumn{5}{|l|}{ Gender } \\
\hline Male & 44 (50.6\%) & $74(43.8)$ & $118(46.1)$ & 0.302 \\
\hline Female & $43(49.4)$ & $95(56.2)$ & 138 (53.9) & \\
\hline \multicolumn{5}{|l|}{ Family members } \\
\hline $1-2$ & $14(16.1)$ & $21(12.4)$ & 35 (13.7) & 0.097 \\
\hline $3-4$ & 24 (27.6) & $27(16.0)$ & $51(19.9)$ & \\
\hline $5-6$ & $22(25.3)$ & $58(34.3)$ & 80 (31.3) & \\
\hline $7-8$ & $12(13.8)$ & $20(11.8)$ & $32(12.5)$ & \\
\hline $9+$ & $15(17.2)$ & $43(25.4)$ & $58(22.7)$ & \\
\hline Employment status & & & & 0.369 \\
\hline Not employed & $56(64.4)$ & 99 (58.6) & $155(60.5)$ & \\
\hline Employed & $31(35.6)$ & $70(41.4)$ & $101(39.5)$ & \\
\hline Chronic disease & & & & 0.671 \\
\hline No & $47(54.0)$ & $96(56.8)$ & $96(56.8)$ & \\
\hline Yes & $40(46.0)$ & $73(43.2)$ & $113(44.1)$ & \\
\hline Hypertension & $22(25.3)$ & $29(17.2)$ & $51(19.9)$ & 0.123 \\
\hline DM & 18(20.7) & $22(13)$ & $40(15.6)$ & 0.11 \\
\hline Dyslipidemia & $15(17.2)$ & $15(8.9)$ & $30(11.7)$ & $0.049^{\star}$ \\
\hline Heart disease & $6(6.9)$ & $6(3.6)$ & $12(4.7)$ & 0.230 \\
\hline Respiratory & $5(5.7)$ & $16(9.5)$ & $21(8.2)$ & 0.304 \\
\hline Renal & $3(3.4)$ & $4(2.4)$ & $7(2.7)$ & 0.615 \\
\hline Hepatic & $2(2.3)$ & $2(1.2)$ & $4(1.6)$ & 0.495 \\
\hline Other & $13(14.9)$ & $25(14.8)$ & $38(14.8)$ & 0.975 \\
\hline Obesity (yes) & $5(5.7)$ & $10(5.9)$ & $15(5.9)$ & 0.956 \\
\hline Current smoker (yes) & $15(17.2)$ & $42(24.9)$ & $57(22.3)$ & 0.166 \\
\hline
\end{tabular}

${ }^{*}$ Significant, $P<0.05$. The bold values indicates statistically significant.

of non-users were women); other demographic characteristics between exposure groups were broadly similar (Table 1).

The amantadine users group had more patients with comorbidities; $46 \%$ of them have a chronic illness compared to $43.2 \%$ in the control group. Hypertension (25.3 vs. $17.2 \%$ ), diabetes (20.7 vs. $13 \%$ ), heart disease (6.9 vs. $3.6 \%$ ), dyslipidemia (17.2 vs. $8.9 \%$ ), and chronic kidney disease (3.4 vs. $2.4 \%$ ). The clinical data listed in Table $\mathbf{1 .}$

\section{Amantadine Dose in Group I}

Amantadine dose ranges from $100 \mathrm{mg}$ daily to $400 \mathrm{mg}$, with no statistically significant difference in the dose between patients
TABLE 2 | Amantadine doses in group I.

\begin{tabular}{lcccc}
\hline Amantadine & Daily dose $(\mathbf{m g})$ & \multicolumn{2}{c}{ COVID-19 } & Total \\
\cline { 3 - 4 } & & No (N, \%) & Yes (N, \%) & \\
\hline & 100 & $21(25.6)$ & $2(40.0)$ & $23(26.4)$ \\
& 200 & $37(45.1)$ & $1(20.0)$ & $38(43.7)$ \\
& 300 & $19(23.2)$ & $1(20.0)$ & $20(23.0)$ \\
& 400 & $5(6.1)$ & $1(20.0)$ & $6(6.9)$ \\
\hline Total & & $82(100)$ & $5(100)$ & $87(100)$ \\
Mean \pm SD & $2.1 \pm 0.9$ & $2.2 \pm 1.3$ & \\
\hline
\end{tabular}

$t=0.252, P=0.801$.

TABLE 3 | COVID-19 risk factors, symptoms and their severity, and PCR results.

\begin{tabular}{|c|c|c|c|}
\hline & $\begin{array}{c}\text { Group I } \\
N(\%)\end{array}$ & $\begin{array}{c}\text { Group II } \\
N(\%)\end{array}$ & $P$ \\
\hline Adopting precautions & & & 0.671 \\
\hline No & $13(14.9)$ & $22(13.0)$ & \\
\hline Yes & $74(85.1)$ & $147(87.0)$ & \\
\hline History of travel & & & 0.932 \\
\hline No & $79(90.8)$ & $154(91.1)$ & \\
\hline Yes & $8(9.2)$ & $15(8.9)$ & \\
\hline Contact with a case & & & 0.469 \\
\hline No & $65(74.7)$ & $119(70.4)$ & \\
\hline Yes & $22(25.3)$ & $50(29.6)$ & \\
\hline COVID-19 symptoms & & & 0.916 \\
\hline No & $71(81.6)$ & $137(81.1)$ & \\
\hline Yes & $16(18.4)$ & 32 (18.9) & \\
\hline Swab for SARS CoV2 & & & 0.299 \\
\hline Not done & $52(59.8)$ & $94(55.6)$ & \\
\hline Negative & $30(34.5)$ & $55(32.5)$ & \\
\hline Positive & $5(5.7)$ & $20(11.8)$ & \\
\hline Symptomatic & $5(100.0)$ & $17(85.0)$ & 1.00 \\
\hline Hospitalized & $2(40.0)$ & $5(25.0)$ & 0.597 \\
\hline ICU & $0(0.0)$ & $1(5.0)$ & 1.00 \\
\hline Ventilation & $0(0.0)$ & $1(5.0)$ & 1.00 \\
\hline Oxygen use & $1(20.0)$ & $7(35.0)$ & 0.643 \\
\hline
\end{tabular}

infected with COVID compared with those who were not. Dose mean, SD (2.1 \pm 0.9 vs. $2.2 \pm 1.3$ ) (Table 2$)$.

\section{COVID-19 Infection and Related Risk Factors}

Regarding the COVID-19 symptoms, almost the same percentage of patients in both groups have symptoms suggestive of COVID19 infection ( 18.9 vs. $18.4 \%$ ). $40.2 \%$ of the total number of patients in group I underwent PCR testing for COVID-19(5.7\% of them have positive results) in comparison to $44.3 \%$ of patients in group II (11.8\% of them resulted as positive) (Table 3 ).

There was no statistically significant difference between both groups regarding different known risk factors of COVID-19 
TABLE 4 | Sequence of COVID infection on different neurologic symptoms.

\begin{tabular}{lccc}
\hline $\begin{array}{l}\text { Effect of COVID } \\
\text { infection }\end{array}$ & Group I & Group II & $\boldsymbol{P}$ \\
\hline $\begin{array}{l}\text { Worsening of } \\
\text { baseline neurological } \\
\text { disease }\end{array}$ & $0(0.0)$ & $9(45.0)$ & 0.123 \\
$\begin{array}{l}\text { Worsened memory } \\
\text { Worsened mood }\end{array}$ & $1(20.0)$ & $7(35.0)$ & 1.00 \\
\hline
\end{tabular}

TABLE 5 | Odds ratio and its 95\% confidence limits of COVID-19 predictors using forced entry multiple logistic regression model.

\begin{tabular}{lrccc}
\hline Predictor & $\boldsymbol{\beta}$ & $\boldsymbol{P}$ & OR & $\mathbf{9 5 \%} \mathbf{C l}$ of OR \\
\hline Age by 10y & 0.047 & $\mathbf{0 . 0 2 8 ^ { * }}$ & 1.048 & $1.005,1.092$ \\
Gender & -0.237 & 0.665 & 0.789 & $0.270,2.304$ \\
Nationality & 0.406 & 0.491 & 1.501 & $0.472,4.766$ \\
Family members & 0.079 & 0.171 & 1.082 & $0.967,1.211$ \\
Work & 0.237 & 0.671 & 1.267 & $0.424,3.786$ \\
Smoking & -0.127 & 0.843 & 0.881 & $0.250,3.100$ \\
Chronic disease & -1.963 & $\mathbf{0 . 0 0 3}^{\star}$ & 0.140 & $0.038,0.516$ \\
Taking precautions & -1.781 & $\mathbf{0 . 0 0 6}$ & 0.168 & $0.047,0.598$ \\
History of contact & 2.835 & $<\mathbf{0 . 0 0 1}^{*}$ & 17.025 & $5.348,54.199$ \\
Using amantadine & -1.362 & $\mathbf{0 . 0 3 2 ^ { * }}$ & 0.256 & $0.074,0.888$ \\
Constant & -4.107 & $0.005^{\star}$ & - & - \\
\hline
\end{tabular}

${ }^{*}$ Significant, $P<0.05$. The bold values indicates statistically significant.

infection (contact with a case, history of travel, Adopting precautions) (Table 3).

Between March 1, 2020, and March 1, 2021, there were 25 COVID-19 infections (diagnosed by PCR testing) among people with PD or MS, 5 (20\%) of which were among regular users of amantadine. Severe symptoms in the form of (hospitalized, needed ICU, those on a ventilator, needed oxygen most of the time) were noted more in the non-users, although not reaching a statistical significance. The clinical data showed in Table 3.

\section{Post COVID Infection Period}

Worsening of the baseline neurological disease was noted in $45 \%$ of positive patients in group II, with no worsening noted among COVID patients in amantadine users. However, this difference does not reach a statistical significance. Furthermore, we reported worsened memory and mood in both groups, with no statistically significant difference between the two groups (Table 4).

\section{Associations of Outcome With Amantadine Exposure}

\section{Risk Factors for COVID-19 Infection}

Older age and history of contact with COVID case were each associated with increased odds of COVID-19 in multivariableadjusted models as follows: (OR: 1.048; 95\% CI: 1.005, 1.092), (OR: 17.025; 95\% CI 5.348, 54.199), respectively (Table 5).

Amantadine was associated with a significantly reduced risk of COVID-19 disease infection after adjustment for several covariates, including age, sex, and other risk factors (adjusted OR 0.256, 95\% CI 0.074-0.888). Similarly, taking precautions was associated with a significantly reduced risk of COVID-19 disease (OR: 0.168; 95\% CI: 0.047, 0.598) (Table 5).

\section{DISCUSSION}

\section{Summary of Key Results}

In our hospital-based retrospective study, we assessed risk factors for COVID-19 among a cohort of patients known to have PD or MS and its relation to the use of amantadine.

Consistent with existing evidence, individuals who have a history of contact with COVID-19 cases had a higher risk of being diagnosed with SARS-CoV-2 (13). In addition, older people have a higher COVID-19 risk (14). Amantadine prescriptions were associated with a reduced risk of COVID-19 PCR positive cases adjusted for a wide area of demographic factors, potential comorbidities, and other medication. We found a shred of evidence that pre-exposure use of amantadine was associated with a lower risk of COVID-19 infection.

\section{Comparisons With the Literature}

Several studies assessed the anti-inflammatory effects of amantadine $(15,16)$. However, to date, published data reported associations between chronic medication with amantadine and COVID-19 infections are limited to clinical observations. In this respect, a preliminary report by Rejdak and Grieb involved 15 patients with $\mathrm{PD}, \mathrm{MS}$ with previous exposure to amantadine at least 3 months previous to involvement in the study. All these amantadine users had contact with confirmed COVID-19 patients. However, they remained asymptomatic even after they had tested positive for coronavirus (8). Another observational open-label study involving 15 patients supported the protective theory of amantadine, recently performed by Aranda-Abreu et al. (17), who treated patients infected with SARS-CoV-2 using it for 14 days. The presence of clinical symptoms and positivity for IgG antibodies and negativity of IgM against SARS-CoV-2 at the end of the treatment confirmed the diagnosis of SARS-CoV-2 patients infection. Although these patients recovered successfully, treated with other medications along with amantadine. The proposed mechanism is that amantadine can cross the lysosome membrane as a lipophilic molecule and act as an alkalizing agent which will prevent the release of viral RNA into the cell (10). To assess the severity of COVID-19 in Patients suffering from MS and PD, the authors carried out a questionnaire-based study. None of them (PD, $n=5$; MS, $n=$ 10) developed severe clinical manifestations of infectious disease (18). Similarly, in a Community-Based Case-Control study, $8.5 \%$ of PD patients were positive for COVID-19 not medicated with amantadine (19). A case of a 75-year-old woman with PD treated with amantadine and was in direct contact with a positive COVID-19 subject had not had any symptoms related to COVID-19 (20).

Our study has several important strengths. The patient's exposure to medication was not only based on issued prescriptions but was confirmed by asking the patient about actual use, not the one prescribed. Importantly, PCR's results 
were retrieved blindly by an author without access to patients' questionnaires. The long duration of compliance on medication besides retrieval of one-year data is remarkable to strengths as well.

\section{LIMITATIONS}

Several limitations of our study are worth noting. The main is the small cohort of COVID-19 patients. Detailed information on the exact timing of COVID-19 infection is lacking, so we could not incorporate this information into the analyses or assess potential time-varying risk factors or confounding factors.

We have not investigated the relationship between the duration of exposure and the risk of disease in this analysis. There will be an underestimation of total COVID-19 cases (several patients in both groups were not tested for COVID-19) due to the absence of a systematic testing strategy at the start of the pandemic, and a risk of false-negative results as health policy during the study period restricted testing to only COVID-19 patients with severe symptoms or patients before hospitalization for non-COVID-19 indications even in the absence of symptoms suggestive of COVID-19 infection. So, performing a swab or presence of manifestations alone is not a sure marker of suspecting COVID-19 infection and may result in selection bias.

Another limitation is the risk of residual confounding by the use of other medications. In addition, we could not assess the severity of neurological disease that may affect the patient's ability to move and communicate with people; that estimate could be a potential bias. Another important

\section{REFERENCES}

1. Hubsher G, Haider M, Okun MS. Amantadine: the journey from fighting flu to treating Parkinson disease. Neurology. (2012) 78:10969. doi: 10.1212/WNL.0b013e31824e8f0d

2. Kumar B, Asha K, Khanna M, Ronsard L, Meseko CA, Sanicas M. The emerging influenza virus threat: status and new prospects for its therapy and control. Arch Virol. (2018) 163:831-44. doi: 10.1007/s00705-018-3708-y

3. Sawada H, Oeda T, Kuno S, Nomoto M, Yamamoto K, Yamamoto M, et al. Amantadine for dyskinesias in Parkinson's disease: a randomized controlled trial. PLoS ONE. (2010) 5:e15298. doi: 10.1371/journal.pone.0015298

4. Ledinek AH, Sajko MC, Rot U. Evaluating the effects of amantadin, modafinil and acetyl-L-carnitine on fatigue in multiple sclerosis-result of a pilot randomized, blind study. Clin Neurol Neurosurg. (2013) 115:S869. doi: 10.1016/j.clineuro.2013.09.029

5. McKimm-Breschkin JL, Fry AM. Meeting report: 4th ISIRV antiviral group conference: novel antiviral therapies for influenza and other respiratory viruses. Antiviral Res. (2016) 129:21-38. doi: 10.1016/j.antiviral.2016.01.012

6. Cimolai N. Potentially repurposing adamantanes for COVID-19. J Med Virol. (2020) 92:531-2. doi: 10.1002/jmv.25752

7. Tipton PW, Wszolek ZK. What can Parkinson's disease teach us about COVID-19? Neurol Neurochir Polsk. (2020) 54:2046. doi: 10.5603/PJNNS.a2020.0039

8. Rejdak K, Grieb P. Adamantanes might be protective from COVID-19 in patients with neurological diseases: multiple sclerosis, Parkinsonism and cognitive impairment. Mult Scler Relat Disord. (2020) 42:102163. doi: 10.1016/j.msard.2020.102163

9. Torres J, Maheswari U, Parthasarathy K, Ng L, Liu DX, Gong X. Conductance and amantadine binding of a pore formed by a lysine-flanked transmembrane consideration is the potential for exposure misclassification. As with any observational study that uses prescription and dispensed medications' data, we could not accurately capture whether patients took their medicine, causing a potential bias toward null findings. However, we defined exposure based on repeat prescriptions, which is likely to be a good sign of compliance.

\section{DATA AVAILABILITY STATEMENT}

The raw data supporting the conclusions of this article will be made available by the authors upon reasonable request.

\section{ETHICS STATEMENT}

The studies involving human participants were reviewed and approved by the ministry of health IRB, Kuwait. Written informed consent for participation was not required for this study in accordance with the national legislation and the institutional requirements.

\section{AUTHOR CONTRIBUTIONS}

WK: design, questionnaire development, and manuscript writeup. AA: retrieved PCR details and helped in manuscript write-up. JA-H: supervised the project. SE: study idea and theory development. MK: statistical analysis. FA: data collection. All authors contributed to the article and approved the submitted version.

domain of SARS coronavirus envelope protein. Prot Sci. (2007) 16:206571. doi: $10.1110 /$ ps.062730007

10. Smieszek S, Przychodzen B, Polymeropoulos MH. Amantadine disrupts lysosomal gene expression; potential therapy for COVID19. BioRxiv. (2020). doi: $10.1101 / 2020.04 .05 .026187$

11. Fardet L, Petersen I, Nazareth I. Common infections in patients prescribed systemic glucocorticoids in primary care: a population-based cohort study. PLoS Med. (2016) 13:e1002024. doi: 10.1371/journal.pmed.1002024

12. Fitzgerald KC, Mecoli CA, Douglas M, Harris S, Aravidis B, Albayda J, et al. Risk factors for infection and health impacts of the COVID-19 pandemic in people with autoimmune diseases. MedRxiv Preprint. (2021) 2021.02.03.21251069. doi: 10.1101/2021.02.03.21251069

13. Meyerowitz EA, Richterman A, Gandhi RT, Sax PE. Transmission of SARSCoV-2: a review of viral, host, and environmental factors. Ann Intern Med. (2020) 174:169-79. doi: 10.7326/M20-5008

14. Clark A, Jit M, Warren-Gash C, Guthrie B, Wang HH, Mercer SW, et al. Global, regional, and national estimates of the population at increased risk of severe COVID-19 due to underlying health conditions in 2020: a modelling study. Lancet Glob Health. (2020) 8:e1003-17. doi: 10.1016/S2214-109X(20)30264-3

15. Palabiyikoglu M, Ormeci N, Ekiz F, Beyler AR, Erdem H, Dökmeci A, et al. Amantadine in non-responder patients with chronic hepatitis C: a randomized prospective study. Hepatogastroenterology. (2012) 59:19114. doi: 10.5754/hge10341

16. Xing W, Huang P, Lu Y, Zeng W, Zuo Z. Amantadine attenuates sepsisinduced cognitive dysfunction possibly not through inhibiting toll-like receptor 2. J Mol Med. (2018) 96:391-402. doi: 10.1007/s00109-018-1631-z

17. Aranda-Abreu GE, Aranda-Martínez JD, Araújo R, Hernández-Aguilar ME, Herrera-Covarrubias D, Rojas-Durán F. Observational study of people 
infected with SARS-Cov-2, treated with amantadine. Pharmacol Rep. (2020) 72:1538-41. doi: 10.1007/s43440-020-00168-1

18. Grieb P, Swiatkiewicz M, Prus K, Rejdak K. Amantadine for COVID-19. JClin Pharmacol. (2021) 61:412-3. doi: 10.1002/jcph.1802

19. Cilia R, Bonvegna S, Straccia G, Andreasi NG, Elia AE, Romito LM, et al. Effects of COVID-19 on Parkinson's disease clinical features: a community-based case-control study. Mov Disord. (2020) 35:128792. doi: $10.1002 / \mathrm{mds} .28170$

20. Borra AC. Does amantadine have a protective effect against COVID-19? Neurol Neurochir Pols. (2020) 54:284-5. doi: 10.5603/PJNNS.a2020.0041

Conflict of Interest: The authors declare that the research was conducted in the absence of any commercial or financial relationships that could be construed as a potential conflict of interest.
Publisher's Note: All claims expressed in this article are solely those of the authors and do not necessarily represent those of their affiliated organizations, or those of the publisher, the editors and the reviewers. Any product that may be evaluated in this article, or claim that may be made by its manufacturer, is not guaranteed or endorsed by the publisher.

Copyright $\odot 2021$ Kamel, Kamel, Alhasawi, Elmasry, AlHamdan and Al-Hashel. This is an open-access article distributed under the terms of the Creative Commons Attribution License (CC BY). The use, distribution or reproduction in other forums is permitted, provided the original author(s) and the copyright owner(s) are credited and that the original publication in this journal is cited, in accordance with accepted academic practice. No use, distribution or reproduction is permitted which does not comply with these terms. 